\title{
INTERPRETATION OF POLARIMETRIC AND TOMOGRAPHIC SIGNATURES FROM GLACIER SUBSURFACE: THE K-TRANSECT CASE STUDY
}

\author{
Giuseppe Parrella ${ }^{1}$, Georg Fischer ${ }^{1,2}$, Matteo Pardini ${ }^{1}$, Kostas Papathanassiou ${ }^{1}$, Irena Hajnsek ${ }^{1,2}$ \\ ${ }^{1}$ German Aerospace Center (DLR) - Microwaves and Radar Institute, Wessling, Germany \\ ${ }^{2}$ ETH Zurich - Institute of Environmental Engineering, Zurich, Switzerland
}

\begin{abstract}
The need of large scale observations with high temporal frequency has promoted airborne and satellite remote sensing techniques for glaciological applications. In particular, active microwave sensors, such as synthetic aperture radars (SARs), offer all-weather and daylight independent operability which is of great advantage at high latitudes, where extreme environmental conditions and long period of darkness strongly limit other kinds of sensors. Moreover, longer wavelengths allow to penetrate significantly into dry snow and ice, interacting with surface as well as subsurface features. On the one hand, this makes SAR measurements suitable to investigate the subsurface structure of glaciers and ice sheets. On the other hand, the complex interaction of microwaves with the subsurface layers makes the interpretation of SAR measurements challenging. This study investigates the potential of SAR techniques to retrieve information about glacier subsurface. SAR polarimetry and tomography are used to gain a 3-D characterization of the scattering scenario of the K-transect, a site located in the ablation zone of Greenland. For this, a fully-polarimetric tomographic airborne dataset, acquired by the DLR's F-SAR system in the frame of the ARCTIC15 campaign, is exploited.
\end{abstract}

Index Terms - Greenland, Synthetic Aperture Radar, glacier dynamics, ice structure.

\section{INTRODUCTION}

Remote sensing systems provide systematic and frequent observations of the polar regions. Synthetic aperture radars (SARs) are likely the most suitable sensors due to their dayand-night and all-weather operability. A further unique aspect of SARs with respect to glaciers and ice sheets monitoring is the capability of microwaves to penetrate (depending on frequency) into dry snow and ice and interact with subsurface features. This allows SAR measurements to gain sensitivity of surface as well as near-surface structure of ice masses which, in turn, reveal information about their temporal dynamics. However, the backscattered signal depends on many parameters (i.e. snow and ice properties, presence of internal layers, inclusions, etc.) and complicates the retrieval of geophysical parameters.

Polarimetric SAR (PolSAR) is an extension of singlechannel SAR. It is based on the use of differently polarized waves and allows a better characterization of the imaged areas by gaining sensitivity to different scattering mechanisms (volume, surface, etc.). Some studies have addressed the interpretation of PolSAR signatures observed over glaciers and ice sheets by developing dedicated scattering models. In [1] authors modelled C-, L- and P-band backscattering from the percolation zone of Greenland as the sum of contributions from ice lenses and glands, buried in snow. The results were rather good at $\mathrm{C}$-band but poorer at L- and P-band. A three-component model was developed in [2] to explain L- and P-band PolSAR measurements from the Austfonna ice cap, in Svalbard. The total backscattering was modelled as the sum of a surface component generated at a shallow snow-ice interface, a volume contribution from dipole-like ice inclusions, and a component associated to sastrugi at the glacier surface. Despite the higher complexity, this model failed to explain the polarization phase differences observed in the data. More recently, a different model has been proposed to interpret L- and P-band signatures from the same ice cap, where a novel component was able to account for propagation effects due to the presence of firn layers [3]. However, already at this stage, the number of involved model parameters is by far larger and requires a number of assumptions. On the other hand, polarimetry only allows a 2-D mapping of the scattering mechanisms as it does not provide information about the vertical distribution of the scatterers.

To further extend the SAR observation space, polarimetric SAR tomography (Pol-TomoSAR) can be exploited. Such technique is based on multi-baseline (MB) (polarimetric) interferometric measurements and allows characterizing the 3-D properties of scattering volumes. The potential of Pol-TomoSAR for glacier subsurface imaging has been already investigated in a few airborne studies [4], [5], [6], [7]. In [4], P-band polarimetric measurements of two sites located on the K-transect of Greenland revealed the presence of scattering sources down to $60 \mathrm{~m}$ depth. As well, 
some morphological subsurface features could be found at one of the two sites. A second study, investigated the potential of Pol-TomoSAR L-band data to image the subsurface of a glacier in the Austrian Alps [5]. The tomographic analysis revealed that the SAR backscattering is a mixture of surface scattering and a complex pattern of subsurface volume scattering. In the firn area, the signal was concentrated in the top 10-20 m while, in the ablation zone, where the subsurface consists of compact ice, scattering sources were visible at higher depth. In some areas, bedrock reflections down to $50 \mathrm{~m}$ depth could also be observed. The first multi-frequency study was presented in [6] again for the K-transect, exploiting the dataset used also in this paper. The analysis focused on the separation of surface and underlying volume scattering. L-band data revealed the presence of two different types of subsurface; the first one mainly constituted by a $10 \mathrm{~m}-15 \mathrm{~m}$ thick volume located around $20 \mathrm{~m}$ depth, and the second type made of surface-like layers. P-band data show less sensitivity to small scatterers, and surface-like multiple layering seems to be the dominant scattering mechanism. Finally, the reduced penetration of C-band (about $10 \mathrm{~m}$ ) did not allow to map the two different volume types anymore.

Despite the demonstrated potential of tomography for glacier subsurface imaging, the application of TomoSAR for glaciological applications is still in an early stage, essentially due to the limited amount of available experimental and validation data. In particular, the physical link between TomoSAR observables and actual subsurface features is still missing. One of the limiting factors is that tomographic power profiles are insensitive to complex-valued components, such as propagation effects, while coherent pol-TomoSAR processing is not accurate enough to provide a reliable characterization of scattering mechanisms.

This paper presents the analysis of multi-frequency Pol-TomoSAR data acquired over the K-transect, south-west Greenland, in the ablation zone of the ice sheet. The aim is to exploit jointly the capability of tomography to provide the 3-D distribution of the scattering sources, the sensitivity of polarimetry to different scattering mechanisms, and the complementarity of different wavelengths to improve the understanding of the physical link between SAR measurements and glacier subsurface structure.

\section{METHODOLOGY}

This study addresses the interpretation of tomographic profiles obtained at different polarizations and frequencies by integrating the information gained through a polarimetric analysis conducted in parallel on the same data. In particular, the 3-D distribution of the backscattering in the glacier subsurface is investigated by means of tomography. Different frequencies are exploited to characterize scatterers of different size and layers located at different depths. For the polarimetric analysis, a set of descriptors is considered, including the scattering entropy, the mean alpha angle, the co-polarization (co-pol) power ratio and phase difference, as already proposed in [3]. In particular, the co-pol ratio is an indicator of the scatterers' orientation: values $>1$ suggest scattering from horizontal structures, values $\approx 1$ are attributed to a random volume, and values $<1$ indicate scattering from vertical features or from a (slightly rough) surface [6]. The co-pol phase difference provides information about the dielectric properties of the medium and, in particular, it can reveal the presence of anisotropic layers, such as firn and (fresh) snow. The scattering entropy indicates whether a dominant scattering mechanism is present (low entropy), or more mechanisms contribute to the total backscatter (high entropy). Finally, the mean alpha angle can be used to identify the main scattering mechanism.

By exploiting the available polarimetric and tomographic SAR observation space, a complete 3-D characterization of the scattering scenario can potentially be achieved. A physical interpretation will be finally attempted by considering the recent dynamics of the study area reported by glaciological studies.

\section{PRELIMINARY RESULTS}

The test site is the K-transect, south west Greenland, a regularly studied area of the ice sheet, with in-situ surface mass balance measurements going on since the early $1990 \mathrm{~s}$ and a number of glaciological studies concerning the different aspects of its temporal dynamics [8]. The available SAR dataset was collected by the F-SAR airborne system of DLR during the ARCTIC15 campaign, which took place from mid-April to the end of May 2015. Multi-baseline fully-polarimetric acquisitions were performed at P-, L-, S-, $\mathrm{C}$-, and X-band covering an area of about $3 \mathrm{~km} \mathrm{x} 24 \mathrm{~km}$, from the west coast to the inner part of the ablation zone of the ice sheet.

First insights concerning the polarimetric behavior of the study area across frequency can be gained by means of the RGB representation of the data in Figure 1. The top panel shows the optical (GoogleEarth) image of the K-transect, which does not show particular features, except the glacier terminus towards the west coast (left) of Greenland. In contrast, the RGB SAR images appear rather heterogeneous and rich of features at all frequencies. Moving from short (X-band, second panel) to longer wavelengths (P-band, bottom panel), a number of dark spots aligned parallel to the azimuth direction becomes more evident. This might indicate that they are located below the ice surface, at a depth that can be better sensed at low frequencies. On the other hand, the $\mathrm{X}$ - and $\mathrm{C}$-band images show very clearly the high roughness of the ice surface, probably related to the presence of crevasses and supraglacial channels. The dominance of greenish pixels points out strong depolarization at all frequencies. At S-, L- and P-band, this can be attributed to volumetric scattering 

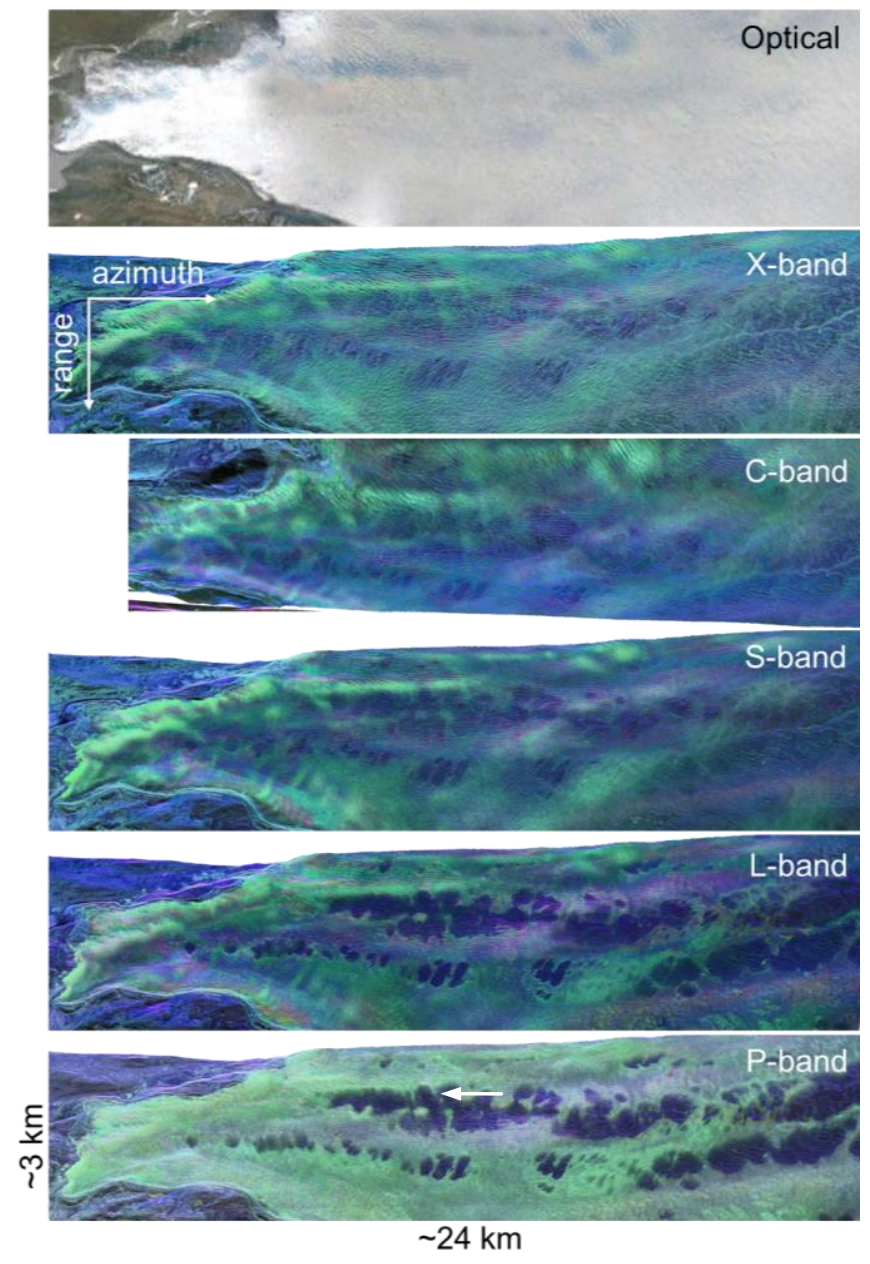

Figure 1. Top panel: optical image of the K-transect; Panel 2-5 (from top): RGB representation of the PolSAR data from X- to P-band (R: HH, G: HV, B: VV). The white arrow indicates the transect considered for preliminary tomographic analysis.

from the subsurface layers. At high frequencies, significant surface roughness might be the reason for depolarized backscattering. Interestingly, the mentioned dark spots show very low depolarization, indicating a clear difference of scattering mechanisms compared to the surrounding areas.

Figure 2 shows first Capon tomographic profiles extracted from a $1 \mathrm{~km}$ transect (white arrow in bottom panel of Figure 1) at HV polarization. A first analysis points out that the dark areas in Figure 1 are characterized by strong scattering at the glacier surface, especially at L- and P-band, while green pixels correspond to areas dominated by subsurface scattering. In agreement with the first polarimetric interpretation, C- and X-band tomograms show that the backscattering at shorter wavelengths is concentrated close to the ice surface.

In the full paper, additional details of the polarimetric and tomographic analysis will be shown and discussed. The results will be interpreted also in the context of the temporal

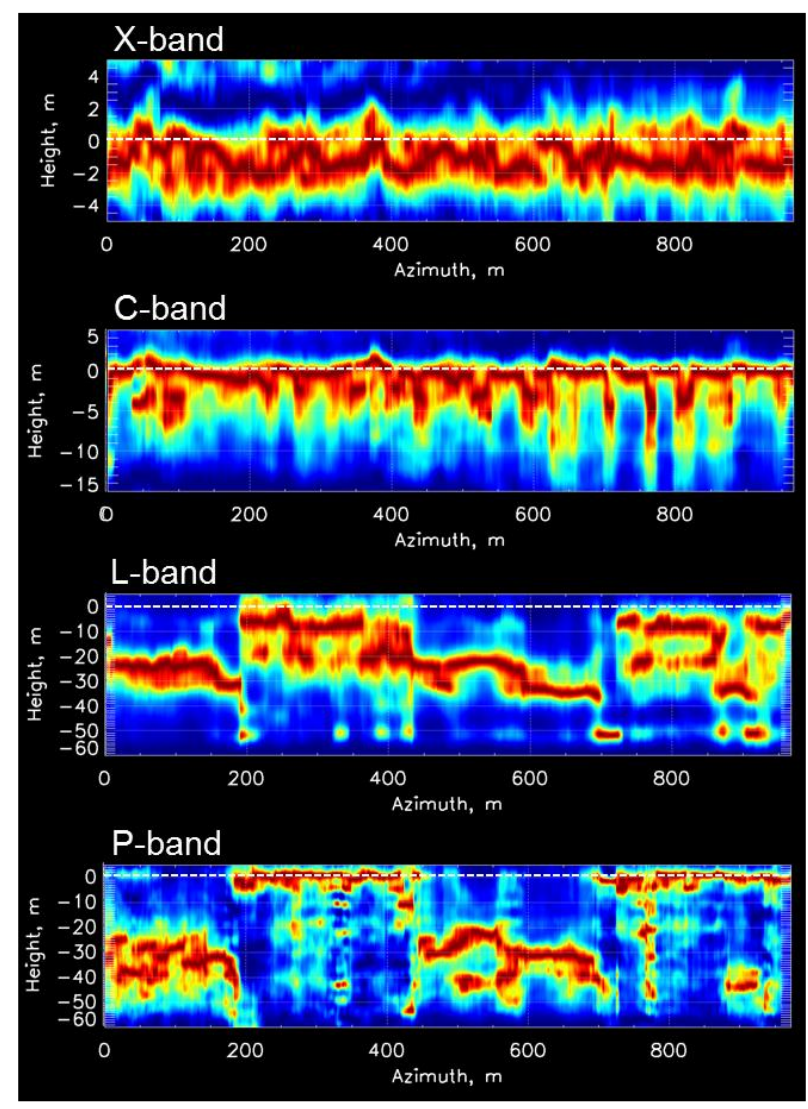

Figure 2. Capon tomograms of the $1 \mathrm{~km}$ transect shown in Fig.1 (white arrow) at HV polarization.

dynamics of the K-transect reported by glaciological studies.

\section{REFERENCES}

[1] E. J. Rignot, "Backscatter model for the unusual radar echoes from the Greenland ice sheet," J. Geophys. Res., 100(E5), 9389-9400, May 1995.

[2] J. J. Sharma, et al., "Polarimetric decomposition over glacier ice using long-wavelength airborne PolSAR," IEEE Trans. Geosci. Remote Sens., 49(1), 519-534, Jan. 2011.

[3] G. Parrella, I. Hajnsek and K. Papathanassiou, "Polarimetric decomposition of L-band PolSAR backscattering over the Austfonna ice cap", IEEE Trans. Geosci. Remote Sens., 54(3), 1267-1281, Mar. 2016.

[4] F. Banda, J. Dall, and S. Tebaldini, "Single and multipolarimetric P-band SAR tomography of subsurface ice structure," IEEE Trans. Geosci. Remote Sens., 54(5), 28322845, May 2016.

[5] S. Tebaldini, T. Nagler, H. Rott, A. Heilig, "Imaging the internal structure of an Alpine glacier via L-band airborne SAR tomography", IEEE Trans. Geosci. Remote Sens., 54(12), 7197-7209, Dec. 2016.

[6] M. Pardini, G. Parrella, G. Fischer, K. Papathanassiou, "A multi-frequency SAR tomographic characterization of subsurface ice volume", in Proc. of EUSAR'16, Berlin, Germany.

[7] G. Fischer, M. Pardini, G. Parrella, K. Papathanassiou, I. Hajnsek, "Cancellation of dominant scattering layers in 
Greenland's subsurface for the characterization of ice volumes", in Proc. of EUSAR'18, Aachen, Germany.

[8] P. Smeeths et al., "The K-transect in west Greenland: automatic weather station data (1993-2016)", Arctic, Antarctic and Alpine Res., 50(1), Apr. 2018, doi: https://doi.org/10.1080/15230430.2017.1420954. 